\title{
Solving the inverse problem for determining the optical characteristics of materials
}

\author{
Konstantin P. Lovetski ${ }^{1}$, Andrey A. Zhukov ${ }^{2}$, Michael V. Paukshto ${ }^{3}$, \\ Leonid A. Sevastianov ${ }^{1}$, Anastasiia A. Tiutiunnik ${ }^{1}$ \\ 1 Peoples' Friendship University of Russia (RUDN University) \\ 6, Miklukho-Maklaya St., Moscow, 117198, Russian Federation \\ ${ }^{2}$ ITL Consulting \\ 16, Olkhovskaya St., bldg. 5, Moscow, 105066, Russian Federation \\ 3 Fibralign Corporation \\ 32930, Alvarado-Niles Rd., Suite 350, Union City, CA 9458\%, USA
}

(received: November 10, 2020; accepted: November 12, 2020)

The paper describes a methodology for determining the optical and physical properties of anisotropic thin film materials. This approach allows in the future designing multilayer thin-film coatings with specified properties. An inverse problem of determining the permittivity tensor and the thickness of a thin film deposited on a glass substrate is formulated. Preliminary information on the belonging of a thin-film coating to a certain class can significantly reduce the computing time and increase the accuracy of determining the permittivity tensor over the entire investigated range of wavelengths and film thickness at the point of reflection and transmission measurement

Depending on the goals, it is possible to formulate and, therefore, solve various inverse problems:

- determination of the permittivity tensor and specification of the thickness of a thick (up to $1 \mathrm{~cm}$ ) substrate, often isotropic;

- determination of the permittivity tensor of a thin isotropic or anisotropic film deposited on a substrate with known optical properties.

The complexity of solving each of the problems is very different and each problem requires its own specific set of measured input data. The ultimate results of solving the inverse problem are verified by comparing the calculated transmission and reflection with those measured for arbitrary angles of incidence and reflection.

Key words and phrases: transmittance, reflectance, refractive indices determination, thin films, multilayers, optical coatings, optical properties

\section{Introduction}

The efficiency of production of existing devices for solid-state micro- and nanoelectronics and successful creation of new ones largely depend on the level of development of the technology for manufacturing layers of various

(C) Lovetski K.P., Zhukov A. A., Paukshto M. V., Sevastianov L.A., Tiutiunnik A. A., 2020 
materials with a thickness from several nanometers to tens of micrometers [1]. The design and manufacture of multilayer structures with desired properties from dielectric and/or metal films requires an accurate knowledge of the optical parameters of each layer [2], [3].

Methods for evaluating the electrophysical parameters of dielectric and semiconductor thin-film materials [4] based on regularized methods [5] for solving inverse problems allow accurate determination of the electrophysical parameters of thin-film semiconductor materials [6]-[8]. It becomes possible to create multilayer structures with predetermined properties [9].

The advantage of non-contact methods, which include spectrophotometric and polarimetric methods, is the possibility to carry out measurements without destroying the material and without changing its properties. When using these methods, the interaction of electromagnetic waves in the optical range with the sample material is considered and the intensities of the transmitted and reflected waves are measured. The obtained intensities can be then used to calculate both optical and geometric parameters of the samples [10]-[12].

The advantage of spectrophotometric measurements is the possibility to determine several parameters using one measuring device and one sample [13]. To determine the thickness, permittivity, and electrical conductivity of nanometer films in layered structures, one can use the results of measurements of the reflection and transmission spectra of the optical radiation interacting with them, provided that the mathematical model of their interaction is known [14], [15].

Finding the electrophysical parameters of layered structures from the reflection and transmission spectra of electromagnetic waves is associated with the need to solve inverse ill-posed problems of electrodynamics.

The developed program "Multilayer" serves both for modeling the transmission of light through multilayer thin-film layered media [16]-[18] and for determining the dielectric (permittivity tensor of anisotropic films) and geometric (film thickness) parameters of various thin-film coatings. The program was created based on many years of experience of collaboration with organizations engaged in the design of thin-film coatings [11] used in the production of liquid crystal displays.

\section{Formulation of extended inverse problem}

Methods described in [19] for description of the transmission of an electromagnetic wave through an optical system are also used in solving the inverse problem for determining the optical characteristics of materials. Let us consider formulation of the inverse problem to determine optical parameters of thin film coating.

Within the framework of the inverse problem, it is required to determine, using data on the transmission $T_{m}\left(\lambda_{i}\right)$ and reflection $R_{m}\left(\lambda_{i}\right)$ for various angles of incidence and various polarizations $(m \in[1, \ldots, n]$, here $n$ is the number of different spectrophotometric measurements for each wavelength $\lambda_{i}, i \in[1, \ldots, p]$ from desired range $\left.\left[\lambda_{\text {beg }}, \lambda_{\text {end }}\right]\right)$, the elements of the permittivity tensor $\varepsilon(\lambda)$ of a homogeneous material in a preset wavelength range $\left[\lambda_{\text {beg }}, \lambda_{\text {end }}\right]$. 
Mathematically, this problem reduces to minimization of the variance functional with respect to the unknown parameter $\varepsilon\left(\lambda_{i}\right)$.

$$
\begin{aligned}
& F(\varepsilon(\lambda))= \\
& =\int_{\lambda_{j}=\lambda_{\text {beg }}}^{\lambda_{\text {end }}}\left(\sum_{i=1}^{n}\left(T_{i}(\lambda)-T_{i}\left(\varepsilon_{j}(\lambda), \lambda\right)\right)^{2}+\sum_{i=1}^{n}\left(R_{i}(\lambda)-R_{i}\left(\varepsilon_{j}(\lambda), \lambda\right)\right)^{2}\right) d \lambda .
\end{aligned}
$$

As was noted above, an additional unknown parameter can be the layer thickness $d$, which has to be especially thoroughly determined for thin films. In such cases, the target functional is written as $F(\varepsilon, d)$.

An important feature of the problem under consideration is that the optical parameters have to be found in a continuous wavelength interval $\left[\lambda_{\text {beg }}, \lambda_{\text {end }}\right]$, rather than at separate points in this interval. Typically, the interval of interest covers the visible spectral range from 400 to $800 \mathrm{~nm}$. The search for parameters defined over a given spectral range implies the requirement that the spectral dependences of these parameters would be smooth functions, which influences the structure of solutions.

Solving the task of minimization [20] of the functional (1) frequently involves procedures requiring considerable computational time. The time consumption can be significantly reduced by taking into account certain special features of the system (isotropic vs. anisotropic materials, thick vs. thin layers), that is, by selecting a proper model of light propagation [21]-[23]. Problems related to the classification of materials are considered in the next section.

Note. From mathematical standpoint, the inverse problem of restoring the parameters of a differential operator belongs to the class of so-called ill-posed problems [5], which implies that small variations in the initial data may lead to large changes in the coefficients that have to be calculated. Such behavior of the solution is called unstable. The problem of reconstruction of the elements of the permittivity tensor $\varepsilon(\lambda)$ is the typical example of an ill-posed problem. The problem of correctness poses additional requirements both to the accuracy of initial data and to the stability of solution algorithms. In order to effectively solve an ill-posed problem, it is also highly desirable to take into account all the information known a priori about the system. Finally, it should be noted that poor (uncertain) initial data on the transmission $T_{m}\left(\lambda_{i}\right)$ and reflection $R_{m}\left(\lambda_{i}\right)$ of light in the system (for example, in the case of a significant level of light scattering) may not allow eliminating all the difficulties related to incorrect formulation of the problem.

\section{Classification of media}

In the adopted approach to formulation of the inverse problem, the properties of materials determined by the permittivity tensor are considered as most important [7], [24], [25]. For the Berreman matrix method [26], special features of a material (isotropic vs. anisotropic) are not of principal significance in solving the direct problem. However, the knowledge about such features may significantly simplify solution of the inverse problem by reducing the number of unknown parameters and by making possible the use of 
simplified computational schemes [27]. In connection with this, we present a classification of the materials of interest [25].

The classification is closely related to the relative values and orientations of the principal axes of the refractive index ellipsoid (figure 1).

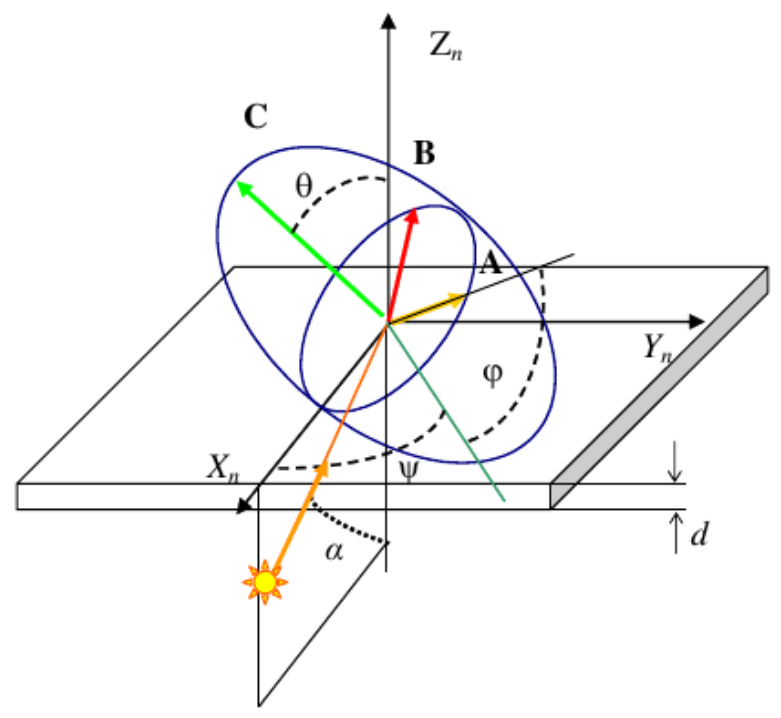

Figure 1. A general orientation of a principal axes of the permittivity ellipsoid. $\theta$ nutation angle, $\psi$ precession angle, $\phi$ spin angle

Classification with respect to the principal values of the permittivity tensor:

- Biaxial anisotropic material. In this case, all three principal axes of the refractive index ellipsoid are different, for example, $\varepsilon_{A}<\varepsilon_{B}<\varepsilon_{C}$. Such materials have two optical axes.

- Uniaxial anisotropic material. Two of the three optical axes are equal to each other, for example, $\varepsilon_{A}=\varepsilon_{B} \neq \varepsilon_{C}$. Such materials have a single optical axis coinciding with $C$ axis.

- Isotropic material. In this case, all three principal axes of the refractive index ellipsoid are equal to each other: $\varepsilon_{A}=\varepsilon_{B}=\varepsilon_{C}$.

In uniaxial materials, directions corresponding to equal principal axes of the refractive index ellipsoid (or equal values of the refractive indices) are called ordinary $\left(\varepsilon_{O}=\varepsilon_{A}=\varepsilon_{B}\right.$ or $\left.n_{o}\right)$, while the remaining direction is called extraordinary $\left(\varepsilon_{e}=\varepsilon_{C}\right.$ or $\left.n_{e}\right)$. It should be recalled that $\varepsilon_{\sigma}=n_{\sigma}^{2}$, where $\varepsilon_{\sigma}, n_{\sigma}$ are complex quantities and $n_{\sigma}$ is the refractive index. Such materials are frequently characterized by the value of birefringence (or double refraction), which is defined as the difference $\Delta n=n_{e}-n_{o}$.

For $n_{e}>n_{o}$ we deal with a positive birefringence, while $n_{e}<n_{o}$ corresponds to a negative birefringence. Now we will consider the commonly accepted classification of uniaxial anisotropic materials with respect to the ratio of $\varepsilon_{O}$ and $\varepsilon_{e}$ and the orientation of a special axis. Here, the main parameter determining the type of a material is the angle of nutation $\theta$.

Classification of uniaxial materials with respect to orientation of the principal axes of the refractive index ellipsoid: 
- o-Plate:

This case corresponds to the general arrangement of a uniaxial refractive index ellipsoid with an angle of nutation within $0<\theta<\pi / 2$, figure 1 .

In cases, when the principal axes of the ellipsoid coincide with the axes of the laboratory frame, the notation $\varepsilon_{A}, \varepsilon_{B}, \varepsilon_{C}$ will be changed to $\varepsilon_{x}, \varepsilon_{y}, \varepsilon_{z}$.

- C-Plate:

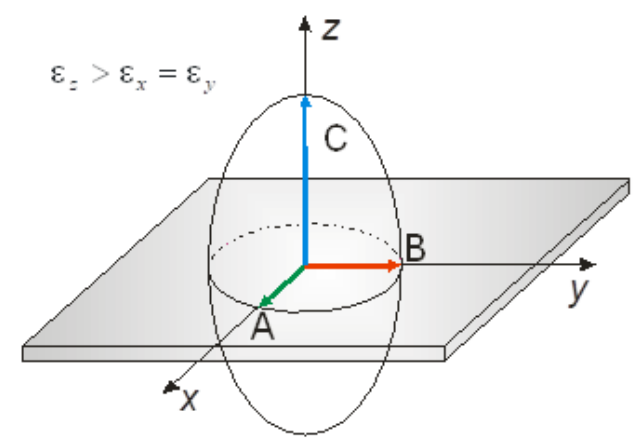

(a) C-plate positive

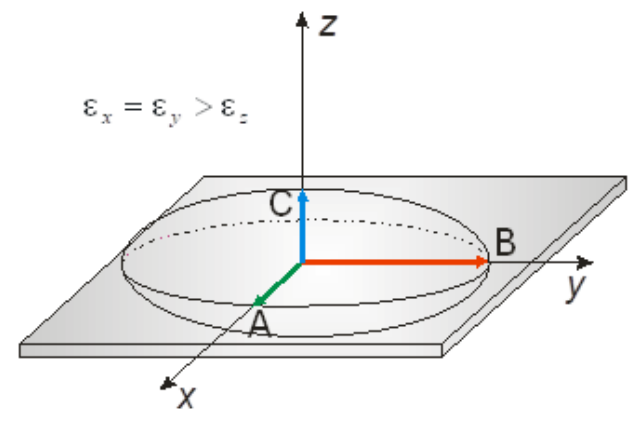

(b) C-plate negative

Figure 2. $[\theta=0 ; \quad \psi=0 ; \psi$ (the angle of rotation measured from $O X$ axis) has arbitrary value

- A-Plate:

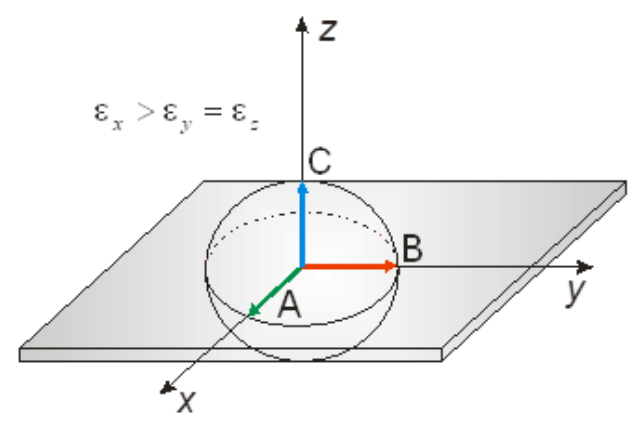

(a) Positive A-plate

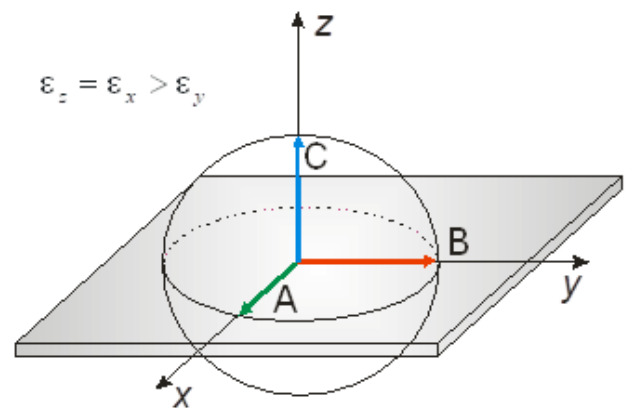

(b) Positive A-plate

Figure 3. A-plate (positive): $\theta=\pi / 2 ; \psi=0 ; \varphi=0$, A-plate (negative):

$$
\theta=\pi / 2 ; \psi=\pi ; \varphi=0
$$

The above classification of uniaxial materials, depending on the orientation of the principal axes of the refractive index ellipsoid of the samples, allows the calculations to be limited to a small number of options for choosing calculation schemes, provided that there is a preliminary information about the belonging of the material under study to one of the classes. 


\section{Classification of inverse problems}

In solving the inverse problem, the material should be classified primarily with respect to two factors: anisotropy and thickness. It should be also taken into account that thin materials (with a thickness of several microns and below) cannot be considered without a substrate bearing this layer. In such cases, calculations of the properties of a thin layer will involve the parameters (refractive index and thickness) of the substrate.

In accordance with a scheme presented in figure 4, the inverse problems are classified as follows.

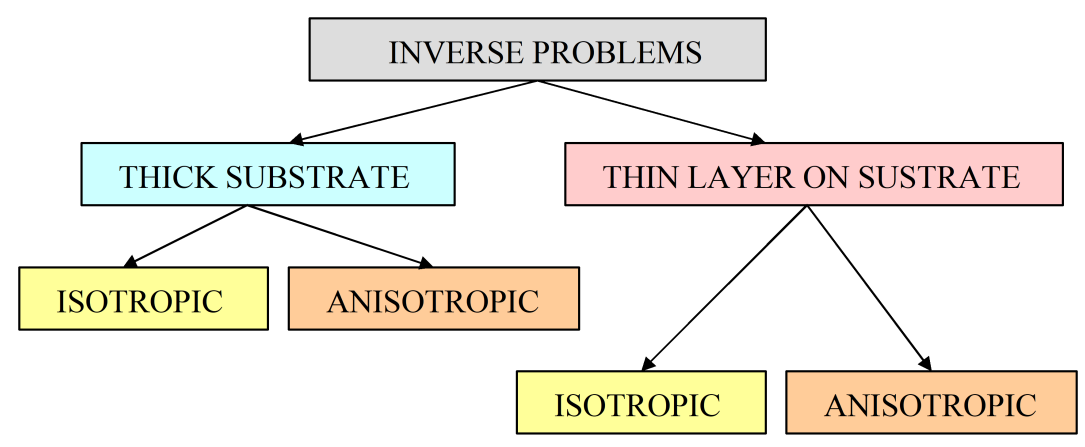

Figure 4. Classification of inverse problems

\section{Problems of determination of refractive indices}

Calculation of optical parameters for a thick layer - usually substrate (onelayer model).

- Calculation of the optical parameters (refractive indices) of an isotropic substrate.

- Calculation of the effective optical parameters of an anisotropic substrate for a given direction in the plane.

- Calculation of the optical parameters (permittivity tensor) of an anisotropic substrate.

Calculation of optical parameters for a thin film on thick substrate (two-layer model).

- Calculation of the optical parameters of a thin isotropic film on isotropic substrate with known parameters.

- Calculation of the effective optical parameters of thin anisotropic film on isotropic substrate with known parameters, for a given direction in the plane.

- Calculation of the optical parameters (permittivity tensor) of a thin anisotropic film on isotropic substrate with known parameters.

The problem of calculation of the effective refractive index of an anisotropic material in a given direction on the plane is solved using the same method as that used for determining the parameters of an isotropic material. However, calculations of the effective refractive index in a certain direction, while being of independent interest, may also perform an auxiliary role. On the one 
hand, the effective refractive indices calculated in two mutually perpendicular directions (par/per) quite well characterize the given material for the normal incidence of light.

On the other hand, calculation of the principal axes of the refractive index ellipsoid for an anisotropic material in the case of small Euler angles (e.g., for a material with well oriented structure, where deviations of the principal axes from axes of the laboratory coordinate system are small) can start with calculation of the components lying in the sample plane. These par/per components can be used in subsequent calculations as very good initial approximation for the complete calculation of the permittivity tensor.

It should be borne in mind that the inverse problems of various types require using different sets of initial data, which will be considered below.

Note. In present version of the program, calculations of the parameters of a thin layer on substrate can be performed only for an isotropic substrate.

\section{Classification of solution structures}

Various methods of calculation of the propagation of light [22], [28] described previously (matrix and classical methods) are based on the relations valid for a given wavelength. In this context, calculation of the spectrum of the refractive index can be performed either point wise (in a given wavelength interval) or using a certain preset parametric dependence (figure 5).

Both point wise and parametric methods of calculation have their own advantages and drawbacks.

\section{Pointwise method}

Advantages. Provides effective solution of the inverse problem in the case of calculations of the optical parameters of a single thick layer. The solution is sufficiently stable. The level of oscillations in the obtained solution (noise) corresponds to the noise level in the initial data. Requires relatively small computational time.

Drawbacks. Gives highly unstable solutions in the case of calculations of the optical parameters of a thin layer in a two-layer structure (strongly ill-posed problem). Satisfactory solution can be obtained using various additional (physically justified) restrictions and regularization parameters.

\section{Parametric structure of solutions}

Advantages. Provides smooth solutions consistent with the physical meaning of the problem. The method is sufficiently universal, especially when the Kramers-Kronig relations are used [29].

Drawbacks. Computational time increases as compared to that required for the point wise calculations. Sometimes it is difficult to select a proper parametric structure of the solution for materials transparent in the entire range $\left[\lambda_{\text {beg }}, \lambda_{\text {end }}\right]$.

It should be noted that the requirement of smoothness is especially important for the real part of the complex permittivity, that is, for the refractive index $(n)$. The spectral dependence of the absorption coefficient $(k)$ is usually smooth even for a pointwise solution.

The main, effective method of solution of the inverse problem consists in obtaining a parametric solution using the Kramers-Kronig relations [29]. In this method, an important step is related to simulation of the absorption peaks. 


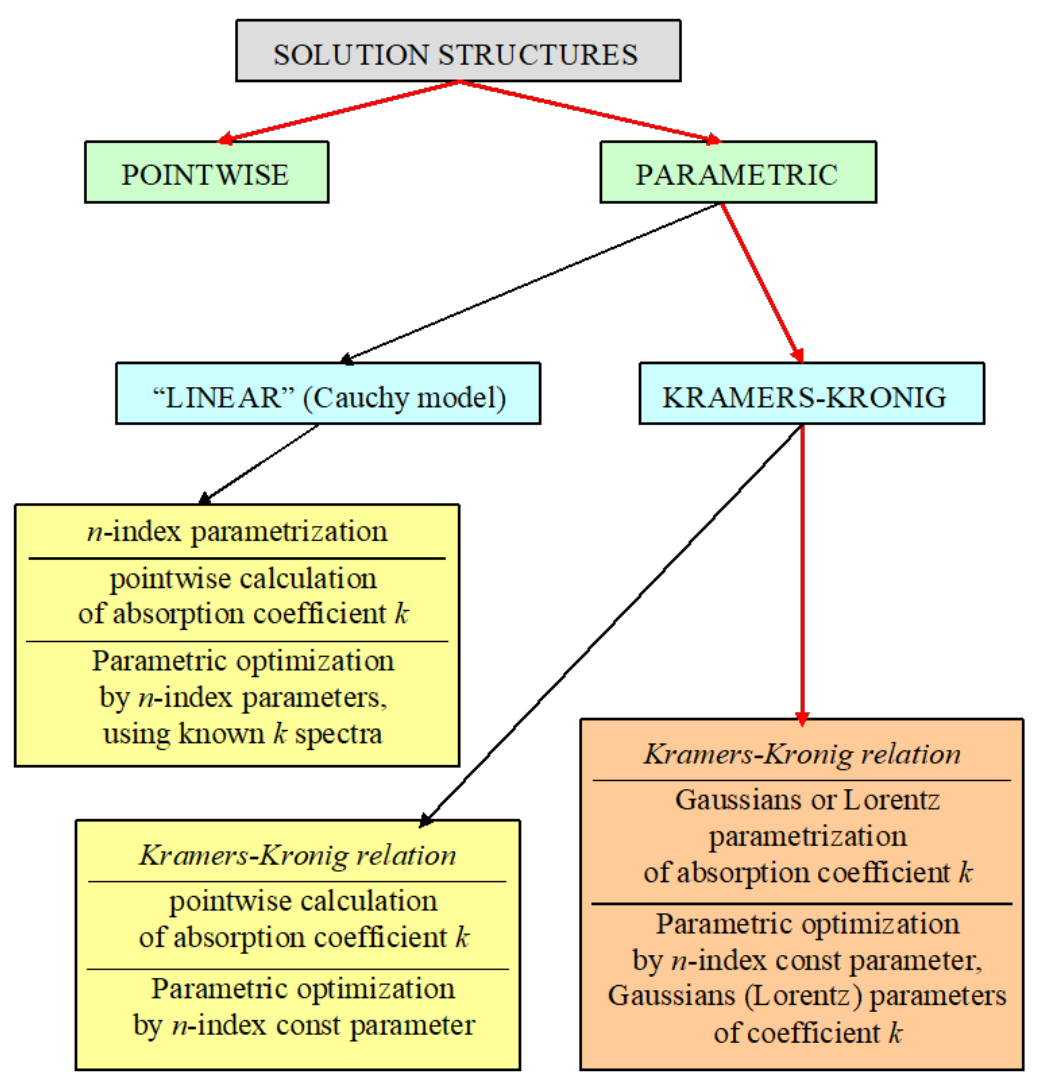

Figure 5. Inverse problems and alternative solution structures. Red field color indicates the most widely used structures

The software "Multilayer" allows the absorption peaks to be approximately described using both Gauss and Lorentz curves. The experience gained in practical calculations shows that the Gauss approximation has to be preferred. Questions pertaining to the Kramers-Kronig relations are considered in more detail in the next Section.

\section{Kramers-Kronig relations for permittivity function}

Assuming that the permittivity function $\left(\varepsilon=\varepsilon^{\prime}+i \varepsilon^{\prime \prime}\right)$ is analytic, we can write the well-known Kramers-Kronig relations between the real $\left(\varepsilon^{\prime}\right)$ and imaginary $\left(\varepsilon^{\prime \prime}\right)$ parts [7]:

$$
\begin{aligned}
& \varepsilon^{\prime}(\omega)-1=\frac{1}{\pi} P \int_{-\infty}^{+\infty} \frac{\varepsilon^{\prime \prime}(x)}{x-\omega} d x, \\
& \varepsilon^{\prime \prime}(\omega)=-\frac{1}{\pi} P \int_{-\infty}^{+\infty} \frac{\varepsilon^{\prime}(x)-1}{x-\omega} d x,
\end{aligned}
$$


where $\omega$ is the circular frequency and $P$ is a symbol indicating the principal value of the integral.

For our purposes, the most important relation is (2) which, by virtue of the odd character of $\varepsilon^{\prime \prime}(\omega)$ [7], is equivalent to the following expression:

$$
\varepsilon^{\prime}(\omega)-1=\frac{2}{\pi} P \int_{0}^{+\infty} \frac{x \varepsilon^{\prime \prime}(x)}{x^{2}-\omega^{2}} d x
$$

Experimental measurements can provide sufficiently reliable data on the absorption coefficient $k(\omega)$ (within a certain limited spectral interval $\left[\omega_{\text {beg }}, \omega_{\text {end }}\right]$ ), which is related to the complex permittivity and the refractive index $n(\omega)$ by the following expressions:

$$
\varepsilon=(n(\omega)+i k(\omega))^{2} \equiv n(\omega)^{2}-k(\omega)^{2}+2 i n(\omega) k(\omega),
$$

or

$$
\varepsilon^{\prime}(\omega)=n(\omega)^{2}-k(\omega)^{2}, \quad \varepsilon^{\prime \prime}(\omega)=2 n(\omega) k(\omega),
$$

where the absorption coefficient $k$ is a dimensionless quantity.

Using equations (4) and (3), one can readily obtain an expression relating the refractive index at a given frequency to the spectrum of the absorption coefficient:

$$
n(\omega)^{2}-k(\omega)^{2}-1=\frac{2 c}{\pi} P \int_{0}^{+\infty} \frac{x n(x) k(x)}{x^{2}-\omega^{2}} d x .
$$

Thus, the main problem in determining the spectral dependence of the refractive index proceeding from the measured absorption spectrum reduces to solving an integral equation (3) with respect to $n(\omega)$. In practice, the absorption spectrum is measured in a certain limited spectral interval $\left[\omega_{1}, \omega_{2}\right]$. In such cases, equation (5) can be rewritten as

$$
n(\omega)^{2}-k(\omega)^{2}-1=\frac{2 c}{\pi} P \int_{\omega_{-b e g}}^{\omega_{-} \text {end }} \frac{x n(x) k(x)}{x^{2}-\omega^{2}} d x+C,
$$

where an error arising on the passage to a finite integration interval is represented by a constant $C$, which, generally speaking, also has to be calculated from experimental data.

Let us consider the results of numerical solution of equation (6) for several model systems, where the absorption coefficients $k$ are defined by a certain set of Gauss functions (Gaussians).

In figure 6 curves drawn in the same type of lines refer to the absorption coefficients (thin lines) and the corresponding refractive index profiles (thick lines) representing solutions of the nonlinear integral equation based on the Kramers-Kronig relations. An interesting example is offered by the pair of curves drawn by dashed lines, which just corresponds to the case under consideration with several absorption bands. The absorption is stronger in 
the initial part of the spectral interval and then decreases. The restored refractive index also exhibits several maxima.

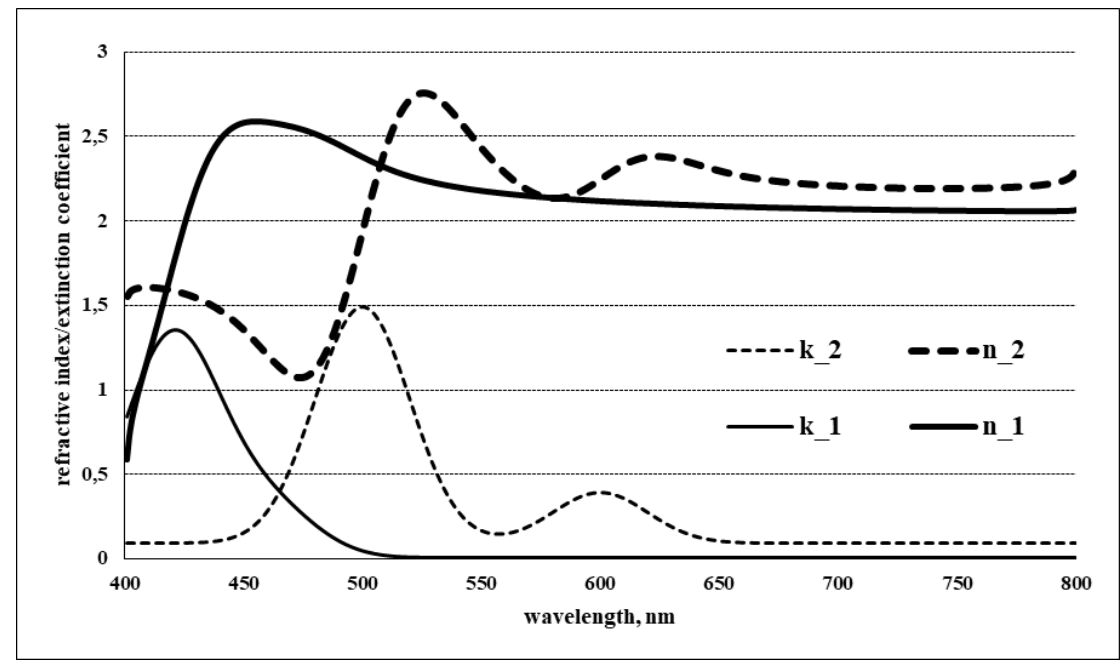

Figure 6. Kramers-Kronig relations. Two solutions $\left(n_{-} 1, k_{-} 1\right)$ and $\left(n_{-} 2, k_{-} 2\right)$ of integral equation (6)

The main approach to solution of the problem of determination of the refractive indices and absorption coefficients using the Kramers-Kronig relations consists in modeling the absorption coefficient by a sum of base functions describing the absorption bands of a given material. Since the spectral interval of measurements $\left[\lambda_{\text {beg }}, \lambda_{\text {end }}\right]$ contains by no means all absorption bands of the given material, it is necessary to provide for the possibility of extending the base functions outside this interval to a wider wavelength range $\left[\lambda_{\text {beg }}^{k}, \lambda_{\text {end }}^{k}\right]$, where $\lambda_{\text {beg }}^{k}<\lambda_{\text {beg }}<\lambda_{\text {end }}<\lambda_{\text {end }}^{k}$. It is assumed that the main absorption bands of the materials under consideration fall within the interval $\left[\lambda_{\text {beg }}, \lambda_{\text {end }}\right]$ or go slightly outside.

The base functions are usually expressed on the frequency scale and then converted to the wavelength scale. For simulations using the developed software, the base functions can be selected in the following forms.

- Gauss functions: $G(\omega, x)=A \cdot \exp \left(-\frac{\left(\omega-\omega_{0}\right)^{2}}{\Delta}\right)$.

— Lorentz functions: $L(\omega, x)=\frac{A}{1+\left(\frac{\omega-\omega_{0}}{\Delta}\right)^{2}}$.

As was noted above, Gaussians are more convenient in use, but Lorentz functions require a shorter time for calculations within each cycle of the iterative process. Use of the Lorentz curves is related to the following disadvantage:

- The shapes of the spectral curves of $n$ and $k$ are less perfect than those obtained by using Gauss functions.

- A significant error arises at the right-hand end of the spectral interval in the course of calculation of the degenerate integral for the refractive index $(n)$. This error can be eliminated by artificially expanding the integration domain (approximately by 40-50 nm) outside the right-hand boundary of the given spectral interval. 


\section{Calculation of optical parameters for a thick layer. One-layer model}

Let us consider some particular examples of calculations of the optical parameters of thick films based on the results of solutions of the corresponding inverse problems.

We'll take as a sample Quartz glass (KU-7 grade) [30].

The transmission and reflection spectra of a sample were measured on a spectrophotometer. The transmission intensity was measured at a zero incidence angle, while the reflection was measured at an angle of $7^{\circ}$ (measured from the normal to the sample surface). The refractive index calculated for the spectral range from 450 to $750 \mathrm{~nm}$ is presented in figure 7. The maximum deviation from the standard is $7.0 \times 10^{-4}$ at a wavelength of $633 \mathrm{~nm}$ (for the curve calculated using the Kramers-Kronig algorithm).

The pointwise algorithm provides a strongly oscillating solution, which is related to insufficient accuracy of the spectral measurements (noisy spectrum) used as the initial data.

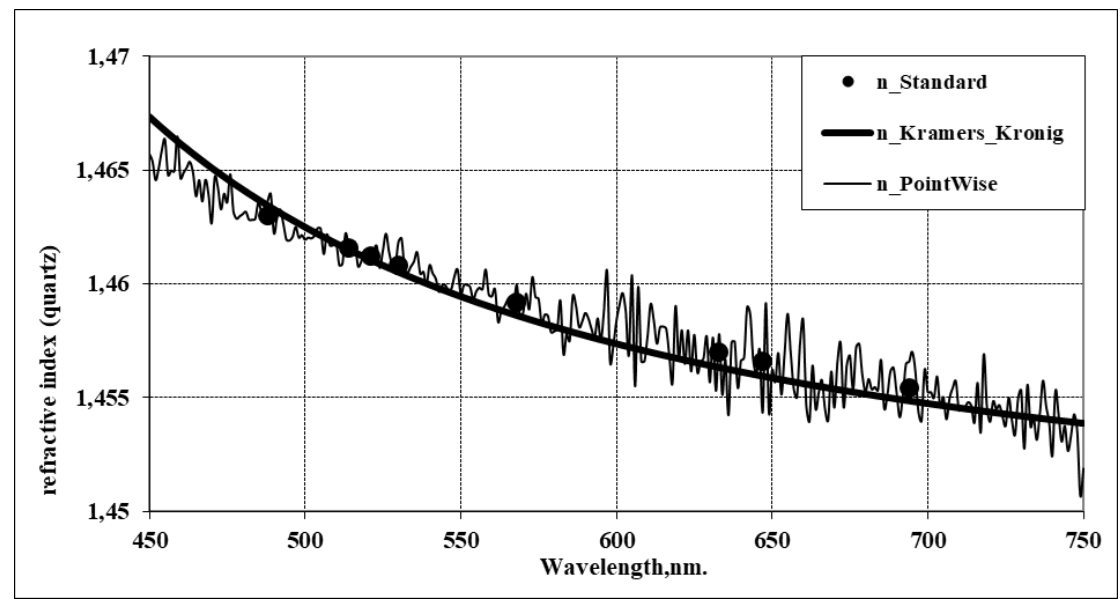

Figure 7. Refractive index of quartz: (Thin) pointwise algorithm; (Thick) Kramers-Kronig algorithm; (Points) standard (tabulated) values

\section{Display glass}

The transmission and reflection spectra of a sample were measured on a spectrophotometer using the same angles of incidence as those in the preceding example - see figures 8 and 9 .

The refractive index calculated for the spectral range from 400 to $800 \mathrm{~nm}$ using the pointwise algorithm and the Kramers-Kronig algorithm (based on the Gaussian approximation of the absorption band) is presented in figure 10 . The spectrum of the absorption coefficient is presented in figure 11.

The pointwise algorithm provides a strongly oscillating solution, which is related to insufficient accuracy of the spectral measurements (noisy spectrum) used as the initial data. It should be also noted that the accuracy of measurements in the beginning and at the end of the spectral interval is reduced because the employed polarizers operate more reliably in the middle of this interval than at the ends. 


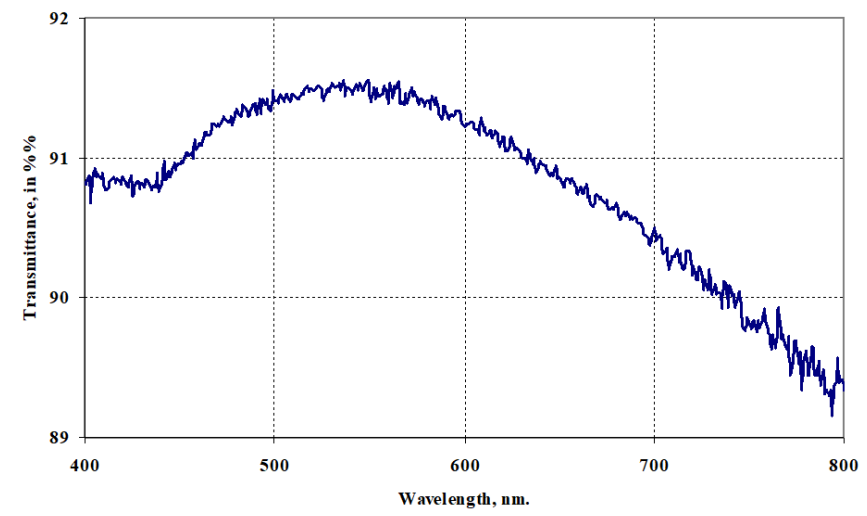

Figure 8. Measured transmission spectra of the glass sample

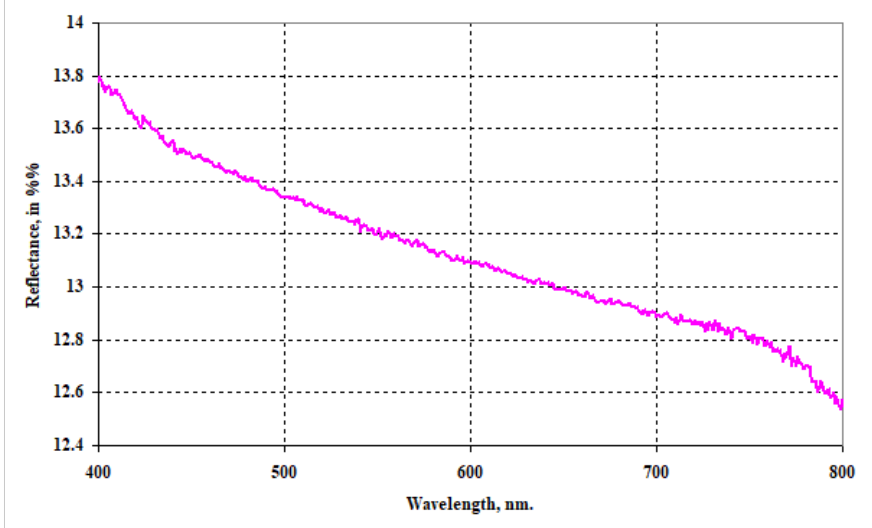

Figure 9. Measured reflection spectra of the glass sample

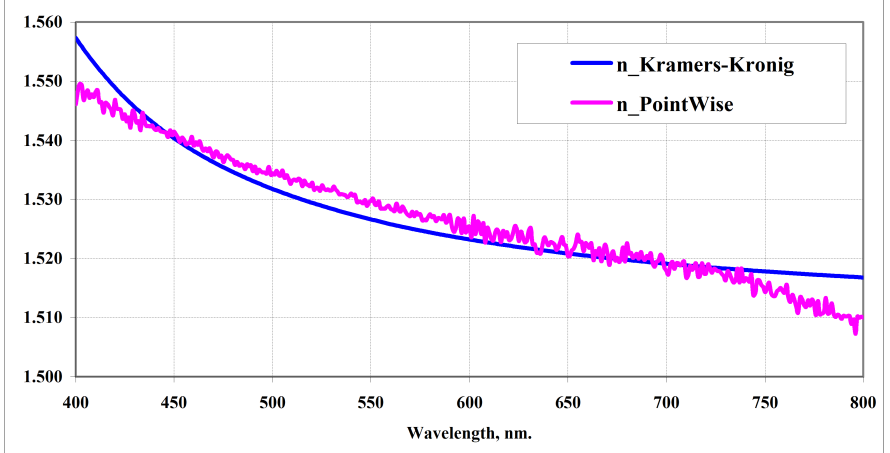

Figure 10. Refractive index of display glass:

(magenta) pointwise algorithm; (blue) Kramers-Kronig algorithm 


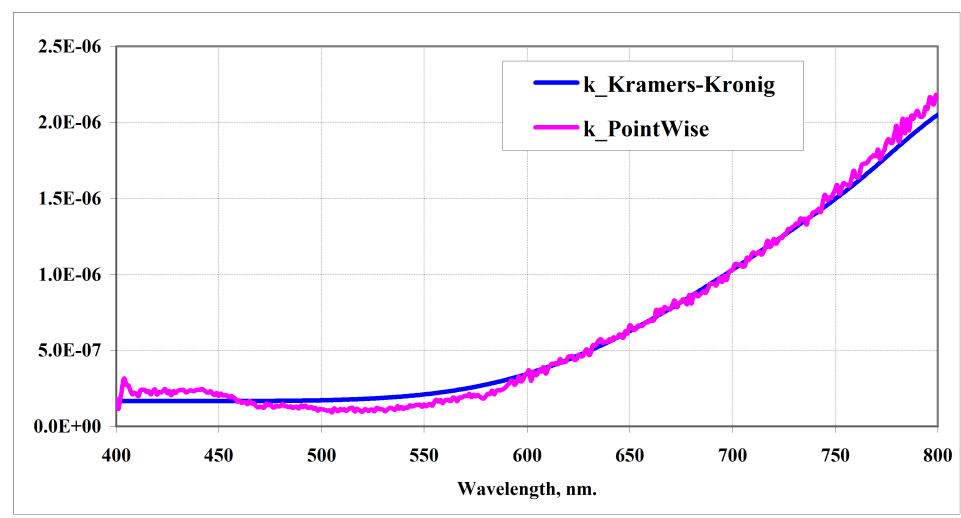

Figure 11. Absorption coefficients of display glass:

(magenta) pointwise algorithm; (blue) Kramers-Kronig algorithm

\section{Calculation of optical parameters for thick and thin layers. Two-layers model}

Calculations of the refractive indices of thin-film materials is complicated by the fact that their transmission and absorption characteristics cannot be measured directly and independently, since such materials are usually deposited onto substrates (with a thickness on the order of $1 \mathrm{~mm}$ ) in the course of fabrication. For this reason, the problem is usually solved in at least two steps. First, it is necessary to determine the parameters of a substrate. Then, the calculated values of the refractive indices and absorption coefficients are used in solving the inverse problem for determining the characteristics of a thin coating.

Let us consider examples of the calculation of refractive indices and absorption coefficients for a tested real thin-film material.

In order to simplify and accelerate the computation procedures, the characteristics are first calculated for a slow direction of a sample. This approach provides sufficiently fast and more accurate evaluation of the film thickness as compared to the standard mechanical measurements with the help of profilometer.

Then, the refractive indices and absorption coefficients are calculated in the perpendicular - fast - direction, with the initial thickness approximation obtained in the first step. After that we can take those solutions as initial ones for solving the problem of index estimation in the third direction - along the vertical axis $O Z$ in local coordinate system. The results of calculation are presented on figures 12 for real parts (refraction indices) and 13 for imaginary parts (absorption coefficients). Figures show the calculated values of principal axes of the refractive index ellipsoid as functions of the wavelength.

Optimization with respect to all components of the refractive index ellipsoid, the Euler angles, and the film thickness at each point of measurements gives the results of fitting for measured transmitted and reflected light depicted in figures $14-15$.

Figures 14 and 15 illustrate the degree of approximation for all eight measurements used in the solution of the inverse problem. The measured and calculated intensities of light transmitted via a thin film deposited onto 
a display glass are compared in figure 14. The intensities of light reflected in various directions for different angles of incidence are presented in figure 15.

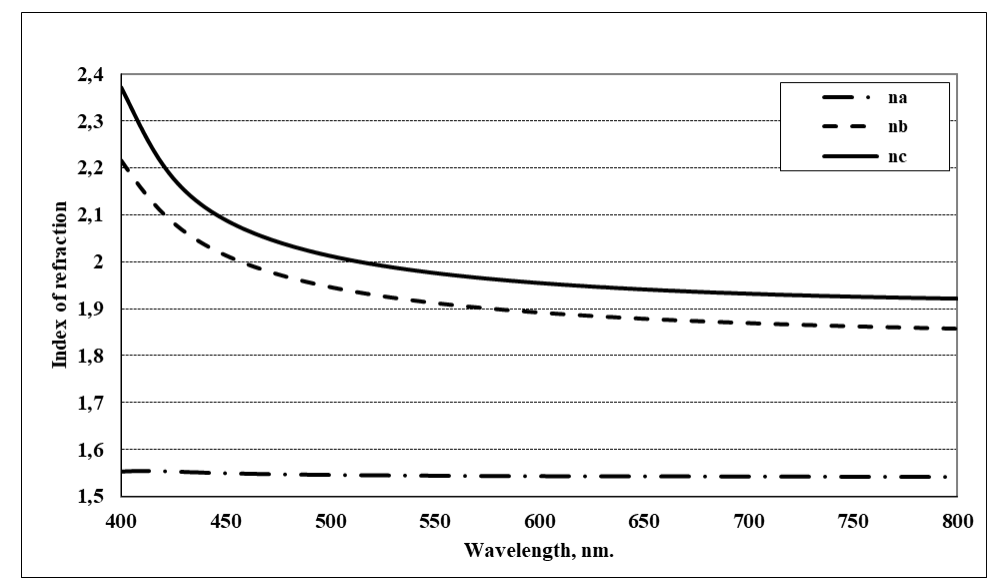

Figure 12. Refractive indices along three axes in local coordinate system

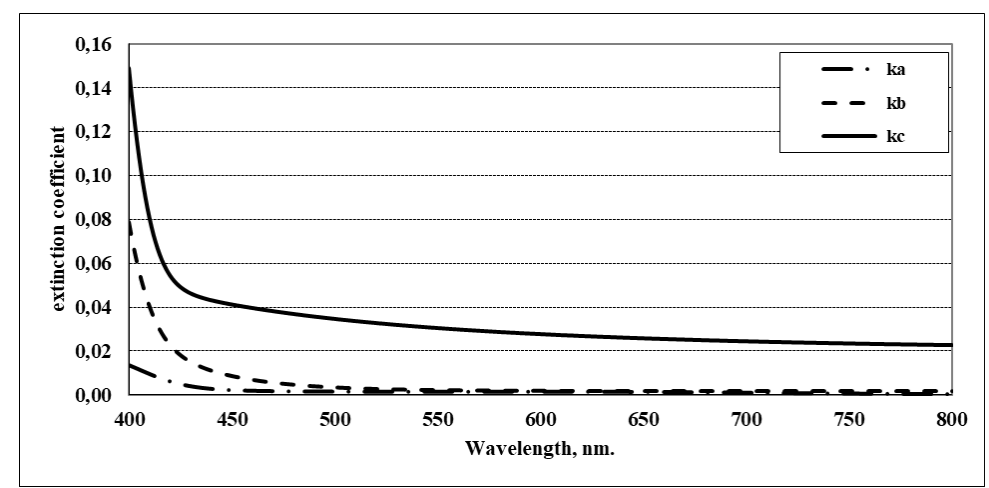

Figure 13. Extinction coefficients along three axes in local coordinate system

It should be noted that the results of fitting with respect to transmission are better than those for reflection, which is related to the fact that the measurements of reflection are more influenced by the scattering of light. Nevertheless, the positions of extrema in the calculated and measured reflection spectra exhibit good coincidence.

\section{Conclusion}

The paper describes a practically tested methodology for the sequential determination of the optical and physical properties (permittivity and thickness) of anisotropic thin-film materials. This approach ensures the determination of the required parameters with high accuracy, which makes it possible in the future to design multilayer thin-film coatings with specified properties. Such coatings can have the properties of absolute mirrors or absolutely black bodies in a given wavelength range, optical filters with desired characteristics, etc. 


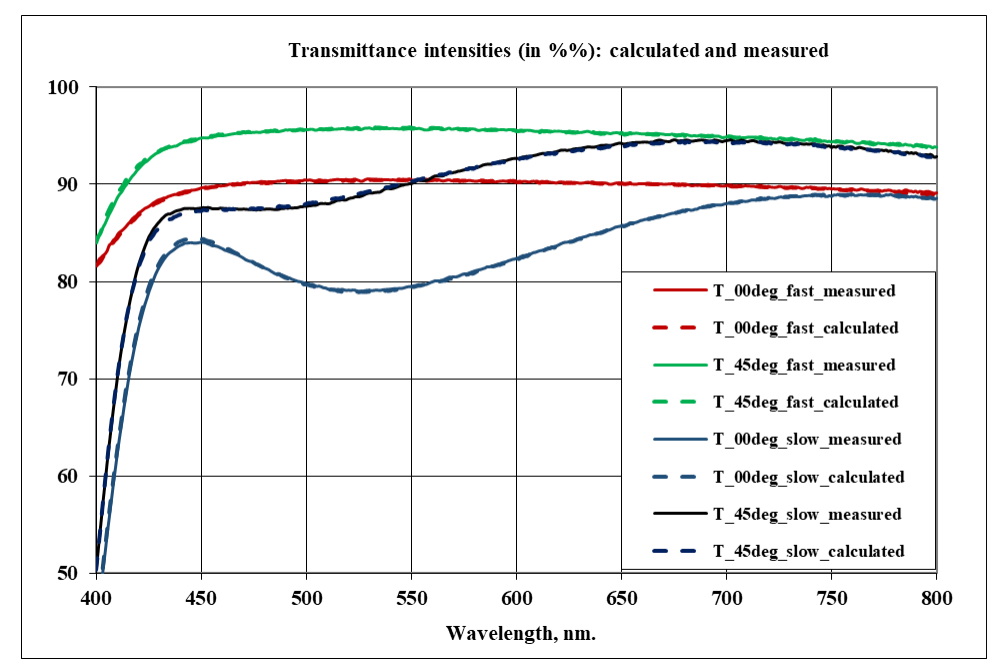

Figure 14. Result of fitting procedure. Calculated (dashed) and measured (solid lines) transmittances for different angles of incident s- and p-polarized light

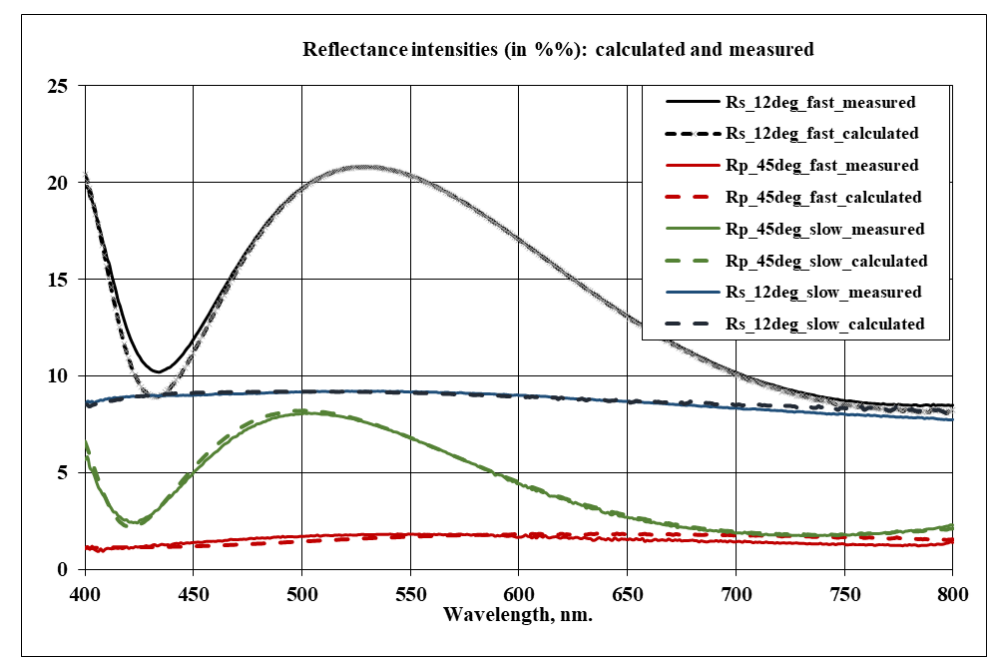

Figure 15. Result of fitting procedure. Calculated (dashed) and measured (solid lines) reflectances for different angles of incident s- and p-polarized light

Depending on the types of the investigated coatings, the user is offered several options (models) for carrying out (executing) calculations. The simplest approach is to solve the inverse problem at each wavelength using the transmission and reflection measured at this wavelength only. In this case, the problem has an (infinite) set of feasible solutions. The choice of the one that is suitable in terms of physical meaning is very difficult for algorithmisation and laborious.

The second approach is to use a priori information on the continuity of the components of the permittivity tensor depending on the wavelength. From a practical point of view, the most successful variant seems to be the approximation of the imaginary part of the tensor by a set of Gaussians (the 
parameters of which are to be determined). The real part of the permittivity tensor is calculated in accordance with the Kramers-Kronig relation.

The complexity of solving each of the problems is very different and each requires its own specific set of measured input data. Therefore, the solution to each problem is implemented as a separate option within the software package, although the solution methodology remains the same.Examples of determining the permittivity of two different standard samples, namely, the KU-7 silica glass and the display glass, from the measured transmission and reflection in the visible range are considered. The obtained values of the refractive index coincide with the values declared by the manufacturer with an accuracy of 4 decimal places.

The efficiency of the method and algorithm for the sequential determination of the permittivity tensor of a thin film deposited on a glass substrate is demonstrated. First, the permittivity of the isotropic substrate is determined and its thickness is refined. At the second stage, after the deposition of a thin anisotropic film on the substrate, the parameters of which must be determined, a series of measurements of transmission and reflection from the two-layer surface is carried out at different angles and in mutually perpendicular directions. The ultimate results of solving the inverse problem are verified by comparing the calculated transmission and reflection with those measured for arbitrary angles of incidence and reflection.

\section{Acknowledgments}

The publication has been prepared with the support of the Russian Foundation for Basic Research (RFBR) according to the research project No 18-07-00567.

\section{References}

[1] D. A. Yakovlev, V. G. Chigrinov, and H. S. Kwok, Modeling and Optimization of LCD Optical Performance. New York: Wiley, 2015.

[2] J. A. Dobrowolski, F. C. Ho, and A. Waldorf, "Determination of optical constants of thin film coating materials based on inverse synthesis," Applied Optics, vol. 22, no. 20, pp. 3191-3200, 1983. DOI: 10.1364/AO. 22.003191.

[3] X. Cheng, B. Fan, J. A. Dobrowolski, L. Wang, and Z. Wang, "Gradientindex optical filter synthesis with controllable and predictable refractive index profiles," Optics Express, vol. 16, no. 4, pp. 2315-3221, 2008. DOI: 10.1364/OE.16.002315.

[4] A. Tejada et al., "Determination of the fundamental absorption and optical bandgap of dielectric thin films from single optical transmittance measurements," Applied Optics, vol. 58, no. 35, pp. 9585-9594, 2019. DOI: $10.1364 / \mathrm{AO} .58 .009585$.

[5] J. B. Bell, A. N. Tikhonov, and V. Y. Arsenin, "Solutions of Ill-Posed Problems," Mathematics of Computation, vol. 32, no. 144, pp. 1320-1322, 1978. 
[6] S. Nevas, F. Manoocheri, E. Ikonen, A. V. Tikhonravov, M. A. Kokarev, and M. K. Trubetskov, "Optical metrology of thin films using highaccuracy spectrophotometric measurements with oblique angles of incidence," in Advances in Optical Thin Films, International Society for Optics and Photonics, vol. 5250, SPIE, 2004, pp. 234-242. DOI: 10.1117/12.512700.

[7] L. D. Landau and E. M. Lifshitz, Electromagnetic Waves in Anisotropic Media. Oxford: Pergamon Press, 1984.

[8] A. V. Tikhonravov et al., "Effect of systematic errors in spectral photometric data on the accuracy of determination of optical parameters of dielectric thin films," Applied Optics, vol. 41, no. 13, pp. 2555-2560, 2002. DOI: $10.1364 / \mathrm{AO} .41 .002555$.

[9] M. Nur-E-Alam, M. M. Rahman, M. K. Basher, M. Vasiliev, and K. Alameh, "Optical and chromaticity properties of metal-dielectric composite-based multilayer thin-film structures prepared by rf magnetron sputtering," Coatings, vol. 10, no. 3, p. 251, 2020. DOI: 10.3390/ coatings 10030251.

[10] S. A. Furman and A. V. Tikhonravov, Basics of optics of multilayer systems. Singapore: World Scientific Publishing, 1992.

[11] M. Paukshto, K. Lovetskiy, and A. Zhukov, "P-59: Dielectric Constants of Display Optical Components," SID Symposium Digest of Technical Papers, vol. 38, no. 1, pp. 410-413, 2007. DOI: 10.1889/1.2785320.

[12] M. Paukshto, K. Lovetsky, A. Zhukov, V. Smirnov, D. Kibalov, and G. King, "P-168: Simulation of Sub-100nm Gratings Incorporated in LCD Stack," SID Symposium Digest of Technical Papers, vol. 37, no. 1, pp. 848-850, 2006. DOI: 10.1889/1.2433649.

[13] A. M. Alsaad et al., "Measurement and ab initio Investigation of Structural, Electronic, Optical, and Mechanical Properties of Sputtered Aluminum Nitride Thin Films," Frontiers in Physics, vol. 8, p. 115, 2020. DOI: $10.3389 /$ fphy. 2020.00115.

[14] R. M. A. Azzam and N. M. Bashara, Ellipsometry and polarized light. Amsterdam: North-Holland Pub. Co., 1977.

[15] Q. M. Al-Bataineh, A. M. Alsaad, A. A. Ahmad, and A. Telfah, "A novel optical model of the experimental transmission spectra of nanocomposite PVC-PS hybrid thin films doped with silica nanoparticles," Heliyon, vol. 6, no. 6, p. 04177 , 2020. DOI: 10.1016/j.heliyon. 2020.e04177.

[16] A. V. Tikhonravov and M. K. Trubetskov. (2020). "OptiChar Software," [Online]. Available: http://www.optilayer.com.

[17] M. Born and E. Wolf, Principles of Optics. London: Pergamon Press, 1980.

[18] T. L. Watkins and J. Fendley, "Refractive index," Physics Education, vol. 18 , no. 2 , p. 56, 1983. DOI: 10.1088/0031-9120/18/2/102. 
[19] K. P. Lovetskiy, N. E. Nikolaev, and A. L. Sevastianov, "Optical Characterization of a Thin-Film Material Based on Light Intensity Measurements," RUDN Journal of Mathematics, Information Sciences and Physics, vol. 26 , no. 3, pp. 252-260, 2018. DOI: $10.22363 / 2312-$ 9735-2018-26-3-252-260.

[20] J. A. Nelder and R. Mead, "A Simplex Method for Function Minimization," The Computer Journal, vol. 7, no. 4, pp. 308-313, 1965. DOI: 10.1093/comjnl/7.4.308.

[21] S.-Y. Lu and R. A. Chipman, "Interpretation of Mueller matrices based on polar decomposition," Journal of the Optical Society of America A, vol. 13, no. 5, pp. 1106-1113, 1996. DOI: 10.1364/JOSAA.13.001106.

[22] P. Yeh, "Extended Jones Matrix Method," Journal of the Optical Society of America, vol. 72 , no. 4, pp. 507-513, 1982. DOI: 10.1364/JOSA.72. 000507.

[23] D. A. Yakovlev and V. G. Chigrinov, "A robust polarization-spectral method for determination of twisted liquid crystal layer parameters," Journal of Applied Physics, vol. 102, no. 2, p. 023 510, 2007. DOI: 10. 1063/1.2756377.

[24] A. Yariv and P. Yeh, Optical Waves in Crystals. New York: John Wiley and Sons, Inc, 2003.

[25] F. I. Fedorov, Optics of Anisotropic Media [Optika anizotropnykh sred]. Minsk: Academy of Sciences of Belarus, 1958, in Russian.

[26] D. W. Berreman, "Optics in Stratified and Anisotropic Media: $4 \times 4$ - Matrix Formulation," The Journal of the Optical Society of America, vol. 62, no. 4, pp. 502-510, 1972.

[27] T. F. Isaev, I. V. Kochikov, D. V. Lukyanenko, A. V. Tikhonravov, and A. G. Yagola, "Comparison of Algorithms for Determining the Thickness of Optical Coatings Online," Computational Mathematics And Mathematical Physics, vol. 59, no. 3, pp. 465-474, 2019. DOI: 10.1134/ S0965542519030102.

[28] J. L. M. Van Mechelen, A. B. Kuzmenko, and H. Merbold, "Stratified dispersive model for material characterization using terahertz timedomain spectroscopy," Optics Letters, vol. 39, no. 13, pp. 3853-3856, 2014. DOI: $10.1364 / 0 \mathrm{~L} .39 .003853$.

[29] A. B. Kuzmenko, "Kramers-Kronig constrained variational analysis of optical spectra," Review of Scientific Instruments, vol. 76, no. 8, pp. 1-9, 2005. DOI: $10.1063 / 1.1979470$.

[30] G. Ghosh, "Refractive Index of Quartz for Thin Film Thickness Measurement," Optics Communications, vol. 163, pp. 95-102, 1999.

\section{For citation:}

K. P. Lovetski, A. A. Zhukov, M. V. Paukshto, L. A. Sevastianov, A. A. Tiutiunnik, Solving the inverse problem for determining the optical characteristics of materials, Discrete and Continuous Models and Applied Computational Science 28 (4) (2020) 378-397. DOI: 10.22363/26584670-2020-28-4-378-397. 
Information about the authors:

Lovetskiy, Konstantin P. - Candidate of Physical and Mathematical Sciences, assistant professor of Department of Applied Probability and Informatics of Peoples' Friendship University of Russia (RUDN University) (e-mail: lovetskiy-kp@rudn.ru, phone: +7(495)9522572, ORCID: https://orcid.org/0000-0002-3645-1060, ResearcherID: A-5725-2017, Scopus Author ID: 18634692900)

Zhukov, Andrey A. - PhD, lead analyst of "ITL Consulting" company (e-mail: a.zhukov@itlc.ru, phone: $+7(495) 6629522$, ORCID: https://orcid.org/0000-0002-4006-2922)

Paukshto, Michael V. - DSc., Physics \& Mechanical Engineering, co-founder and CTO of Fibralign Corporation (e-mail: mpaukshto@fibralignbio.com, phone: 650492 1440, ORCID: https://orcid.org/0000-0002-8090-5402, Scopus Author ID: 6701433110)

Sevastianov, Leonid A. - Doctor of Physical and Mathematical Sciences, professor of Department of Applied Probability and Informatics of Peoples' Friendship University of Russia (RUDN University) (e-mail: sevastianov-la@rudn.ru, phone: +7(495)9522572, ORCID: https://orcid.org/0000-0002-1856-4643, ResearcherID: B-8497-2016, Scopus Author ID: 8783969400)

Tiutiunnik, Anastasiia A. - Candidate of Physical and Mathematical Sciences, lecturer of Department of Applied Probability and Informatics of Peoples' Friendship University of Russia (RUDN University) (e-mail: tyutyunnik-aa@rudn.ru, phone: +7(495)9550783, ORCID: https://orcid.org/0000-0002-4643-327X, ResearcherID: B-8441-2019, Scopus Author ID: 57188573409) 


\title{
Решение обратной задачи определения оптических характеристик материалов
}

\author{
К. П. Ловецкий ${ }^{1}$, А. А. ЖКуков ${ }^{2}$, М. В. Паукшто ${ }^{3}$, \\ Л. А. Севастьянов ${ }^{1}$, А. А. Тютюнник ${ }^{1}$ \\ 1 Российский университет дружбы народов \\ ул. Миклухо-Маклая, д. 6, Москва, 117198, Россия \\ ${ }^{2}$ ITL Consulting \\ Ольховская, д. 16, корп. 5, Москва, 105066, Россия \\ ${ }^{3}$ Fibralign Corporation \\ Альварадо-Найлс Роуд, д. 32930, офис 350, Юнион-Сити, СА 94587, США
}

В работе изложена методология определения оптических и физических свойств анизотропных тонкоплёночных материалов. Такой подход позволяет в дальнейшем проектировать многослойные тонкоплёночные покрытия с заданными свойствами. Сформулирована обратная задача определения тензора диэлектрической проницаемости и толщины тонкой плёнки, нанесённой на стеклянную подложку, с известными оптическими свойствами и толщиной. Предварительная информация о принадлежности тонкоплёночного покрытия к определённому классу позволяет значительно сократить время расчёта и увеличить точность определения тензора диэлектрической проницаемости на всём исследуемом интервале длин волн и толщины плёнки в точке измерения отражения и пропускания.

В зависимости от поставленных целей возможна постановка и, следовательно, решение различных обратных задач:

- определение тензора диэлектрической проницаемости и уточнение толщины толстой (до 1 см) подложки, часто изотропной;

- определение тензора диэлектрической проницаемости тонкой изотропной или анизотропной плёнки, нанесённой на подложку, с известными оптическими свойствами.

Сложность решения каждой из задач весьма различна и каждая требует своего определённого набора измеренных входных данных. Окончательные результаты решения обратной задачи верифицируются с помощью сравнения вычисленных коэффициентов пропускания и отражения с измеренными для произвольных углов падения и отражения.

Ключевые слова: определение коэффициентов пропускания, отражения, показателей преломления, тонкие плёнки, многослойные материалы, оптические покрытия, оптические свойства 